\title{
Index of refraction of noble gases for sodium matter waves
}

\section{Citation}

Forrey, Robert C., Li You, Vasili Kharchenko, and Alex Dalgarno. 1996. "Index of Refraction of Noble Gases for Sodium Matter Waves." Physical Review A 54 (3): 2180-84. https:// doi.org/10.1103/physreva.54.2180.

\section{Permanent link}

http://nrs.harvard.edu/urn-3:HUL.InstRepos:41417406

\section{Terms of Use}

This article was downloaded from Harvard University's DASH repository, and is made available under the terms and conditions applicable to Other Posted Material, as set forth at http:// nrs.harvard.edu/urn-3:HUL.InstRepos:dash.current.terms-of-use\#LAA

\section{Share Your Story}

The Harvard community has made this article openly available.

Please share how this access benefits you. Submit a story.

Accessibility 


\title{
Index of refraction of noble gases for sodium matter waves
}

\author{
Robert C. Forrey, Li You, Vasili Kharchenko, and Alex Dalgarno \\ Institute for Theoretical Atomic and Molecular Physics, Harvard-Smithsonian Center for Astrophysics, 60 Garden Street, \\ Cambridge, Massachusetts 02138
}

(Received 5 December 1995)

\begin{abstract}
We present a theoretical study of the index of refraction of the light noble gases $\mathrm{He}$ and $\mathrm{Ne}$ for the propagation of sodium matter waves. Our approach is based on the well known multiple scattering theory inside a medium, which leads to an approximation for the index of refraction that depends only on the single forward scattering amplitude and the density distribution functions for the atoms of the medium. The forward scattering amplitude between a sodium atom and a noble gas atom is calculated from molecular potential curves using the eikonal approximation. An explicit phase space average is performed over the noble gas atom distribution in the medium. Our results show very good agreement with the recent interferometry measurements of Schmiedmayer et al. [Phys. Rev. Lett. 74, 1043 (1995)]. Our calculations provide an interpretation of the experimental data, and may shed some light on possibilities for obtaining improved interatomic potentials through atomic interferometry measurements. [S1050-2947(96)04709-9]
\end{abstract}

PACS number(s): 03.75.Dg, 03.65.Bz, 07.60.Ly

\section{INTRODUCTION}

Over the past decade significant progress in atomic interferometry research has been made [1]. With its potential for unprecedented accuracy, many believe new fundamental experiments will soon be performed. In a recent experiment, Schmiedmayer et al. [2] have measured the index of refraction of the noble gases for sodium atom matter waves with the MIT interferometer [3], thus opening a new chapter in the application of atom interferometers to atomic physics.

In the experiment [2], a sodium matter wave was coherently split into two parts with a mechanical grating. The two parts were then allowed to propagate along physically separate paths before being coherently recombined for the interference measurement. Since the separation of the two paths of the atom was large enough to neglect the overlap of the wave packets, it was possible for the arm of one path to be filled with a target gas. Thus, the final interference fringes allow a measurement of the additional phase shift to the sodium matter wave introduced by the noble gas medium. For technical reasons, the experiment was most sensitive to the ratio of the real to the imaginary part of the forward scattering amplitude [2]. The measurements can nevertheless be used to determine the effective complex index of refraction of the target gases, and with the application of the multiple scattering theory, they provide the first experiment capable of directly measuring the forward scattering amplitude for sodium passing through the noble gases.

An interpretation based on empirical model potentials was presented in [2], which seems to indicate that the measurements are very sensitive to the shape of the long-range interatomic potential. In this paper, we present a theoretical study of the index of refraction of the light noble gases $\mathrm{He}$ and $\mathrm{Ne}$ for the propagation of sodium matter waves that provides an interpretation that differs from the one presented in [2]. Our approach is based on the well known multiple scattering theory inside a medium [4]. The theory gives an approximation for the index of refraction that depends only on the single forward scattering amplitude and density distribution functions for the atoms of the medium. We use the eikonal approximation [5] to calculate the forward scattering amplitude between the sodium atom and a single noble gas atom, and then perform an explicit average over the phase space distribution of the noble gas atoms inside the medium. We find that for the light target gases, the measurements are most sensitive to the shorter-range "glory" contribution that arises when the scattering phase shift has a maximum as a function of impact parameter. Normally, this maximum phase shift will have a magnitude that is several times the value of $\pi$, and the glory contribution can be estimated using a stationary phase approximation. For the light target gases, however, we find that the maximum phase shift is small compared to $\pi$ and the stationary phase approximation breaks down. When the glory contribution is computed numerically, we find that its magnitude is roughly three times that of the long-range contribution. Therefore, we believe that the interferometry measurements are actually probing the short- to intermediate-range part of the potential for these systems. Since it is in this region where existing molecular potentials are most uncertain, this interpretation points to the possibility of obtaining improved interatomic potentials through use of atomic interferometry measurements.

The paper is organized as follows: In Sec. II, we formulate the theoretical description of the problem in terms of the multiple scattering theory. Section III is devoted to a detailed study of the potential scattering between a sodium atom and a noble gas atom. We also give the details of the molecular potentials that were used. In Sec. IV, the results of the scattering calculations are presented and analyzed. We conclude with a discussion on the possibility of obtaining molecular potential information from the index of refraction measurements.

\section{FORMULATION}

According to the multiple scattering theory [4], the propagation of the sodium matter wave inside a noble gas medium can be characterized by the following coupled equations for the microscopic field $\Psi(\vec{r})$ : 


$$
\begin{gathered}
\Psi(\vec{r})=e^{i \vec{k}_{L} \cdot \vec{r}}+\sum_{i} G\left(\vec{r}-\vec{r}_{i}\right) f_{i} \psi\left(\vec{r}_{i}\right), \\
\psi\left(\vec{r}_{i}\right)=e^{i \vec{k}_{L} \cdot \vec{r}_{i}}+\sum_{j \neq i} G\left(\vec{r}_{j}-\vec{r}_{i}\right) f_{j} \psi\left(\vec{r}_{j}\right),
\end{gathered}
$$

where $e^{i \vec{k}_{L} \cdot \vec{r}}$ is the incident plane wave, and $\psi\left(\vec{r}_{i}\right)$ describes the local field at the site $\vec{r}_{i}$ where the $i$ th noble gas atom is located. $G\left(\vec{r}-\vec{r}_{i}\right)$ is the retarded Green's function $e^{i k_{L}\left|\vec{r}-\vec{r}_{i}\right|} /\left|\vec{r}-\vec{r}_{i}\right|$, which describes the propagation of the scattered wave in the region between the noble gas atoms, while $f_{j}$ is the scattering amplitude. We have assumed the gas to be dilute so that most of the regions between target atoms are in the asymptotic far field region of multiple scatterings. As far as the coherent propagating field inside the medium is concerned, we have to derive the macroscopic field $\langle\Psi(\vec{r})\rangle_{m}$, where the average $\langle\cdots\rangle_{m}$ denotes a coarse graining process. Under the condition for the experiment [2], the linear density of the noble gas atoms is about $\sim 10^{8} / \mathrm{cm}$, and the typical interaction length is about $\sim 10$ $\mathrm{cm}$. The propagation is, therefore, statistical and we can use the optical potential to treat the noble gas as an effective medium with a complex index of refraction $n\left(k_{L}\right)[4,6]$. The solution inside the medium can then be written in the form $\langle\Psi(\vec{r})\rangle_{m} \sim e^{i n \vec{k}_{L} \cdot \vec{r}}$ where

$$
n\left(k_{L}\right)=1+\frac{2 \pi N}{k_{L}}\left\langle\frac{f(k, 0)}{k}\right\rangle,
$$

where $N$ is the number density of the medium and $k_{L}$ is the momentum of the beam in the laboratory frame. The forward scattering amplitude $f(k, 0)$ is calculated in the center of mass frame for two particles with relative momentum $k$. The average $\langle\cdots\rangle$ in (2) refers to a statistical average with respect to all microscopic parameters of the medium. It is convenient to average with respect to the momentum distribution using

$$
\left\langle\frac{f(k, 0)}{k}\right\rangle=\frac{1}{\mu} \int_{0}^{\infty} f(k, 0) \rho(k) d k,
$$

where

$$
\int \rho(k) d\left(\frac{k^{2}}{2 \mu}\right)=1,
$$

and $\mu$ is the reduced mass $m_{T} m_{P} /\left(m_{T}+m_{P}\right)$ with $m_{P}$ and $m_{T}$ the respective masses of the projectile and target atoms. The distribution function is given by

$$
\begin{aligned}
\rho(k)= & \int \delta\left[\frac{\mu\left(\vec{v}_{P}-\vec{v}_{T}\right)^{2}}{2}-\frac{k^{2}}{2 \mu}\right] f_{M}\left(\vec{v}_{T}\right) d \vec{v}_{T} \\
= & \left(m_{T}+m_{P}\right) \sqrt{\frac{\beta}{\pi m_{T} m_{P} E}} \sinh \left(\frac{\beta m_{T} k}{\mu} \sqrt{\frac{2 E}{m_{P}}}\right) \\
& \times \exp \left\{-\beta\left[\frac{k^{2}}{2 \mu}\left(1+\frac{m_{T}}{m_{P}}\right)+E\left(\frac{m_{T}}{m_{P}}\right)\right]\right\},
\end{aligned}
$$

where $E=m_{P} v_{P}^{2} / 2=k_{L}^{2} / 2 m_{P}$ is the kinetic energy of the so- dium atom. We have assumed that the medium of noble gas to be Maxwellian with the distribution of a uniform spatial density $N$,

$$
f_{M}\left(\vec{v}_{T}\right)=\left(\frac{\beta}{2 \pi}\right)^{\frac{3}{2}} \exp \left(-\frac{1}{2} \beta m_{T} v_{T}^{2}\right)
$$

where $\vec{v}_{T}$ is the target velocity, and $\beta=1 /\left(k_{B} T\right)$, with $k_{B}$ the Boltzmann constant, and $T$ the temperature of the noble gas.

In the experiment, the sodium matter wave has a velocity width which is only a few percent of the peak $k_{L}$. Since the relative velocity distribution $\rho(k)$ is much wider than this width, we can neglect the average over the projectile velocity distribution.

\section{POTENTIAL SCATTERING}

The forward scattering amplitude is to be calculated from molecular potential curves between the sodium and the noble gas atoms. We have compared our potential scattering calculations using standard quantum partial wave theory with those using the semiclassical impact parameter treatment and found agreement to within a few percent. Therefore, we will present only the semiclassical theory since it is better suited for our purpose of interpreting the physical contributions to the scattering amplitude. In the eikonal approximation, the scattering amplitude is given by

$$
f(k, 0)=-i k \int_{0}^{\infty}\{\exp [2 i \eta(b)]-1\} b d b,
$$

where $b$ is the classical impact parameter, and $\eta(b)$ is the corresponding phase shift. The scattering amplitude (7) can be divided into two contributions. The first contribution is due to the long-range part of the potential where the phase shift is small. The approximation $\exp [2 i \eta(b)]-1 \approx 2 i \eta(b)$ can therefore be used and the contribution is given by

$$
f_{d}(k, 0)=2 k \int_{b_{1}}^{\infty} \eta(b) b d b
$$

where the impact parameter $b_{1}$ is large enough so that the phase shift in (7) is due to the long-range part of the potential. The subscript ' $d$ ', is often used in (8) to designate diffraction scattering from the tail of the potential [7]. For the long-range interaction potential

$$
V(r)=-\frac{C_{s}}{r^{s}}
$$

the phase shift is [7]

$$
\eta(b)=C_{s} F_{s}\left(\frac{k}{2 E b^{s-1}}\right),
$$

where

$$
F_{s}=\frac{1}{2} \sqrt{\pi} \frac{\Gamma[(s-1) / 2]}{\Gamma(s / 2)} .
$$

The long-range contribution to the scattering amplitude is found to be 


$$
f_{d}(k, 0)=2 k \eta\left(b_{1}\right)\left(\frac{b_{1}^{2}}{s-3}\right)
$$

The second contribution to the scattering amplitude comes from the forward glory scattering and is given by

$$
f_{g}(k, 0)=-i k \int_{0}^{b_{1}}\{\exp [2 i \eta(b)]-1\} b d b .
$$

The term involving -1 is usually integrated and combined with $f_{d}$ since it has the same $b_{1}$ dependence when $\eta\left(b_{1}\right)$ is set equal to a constant. The remaining integral

$$
f_{g}(k, 0)=-i k \int_{0}^{b_{1}} \exp [2 i \eta(b)] b d b
$$

is conventionally calculated by extending the limits of integration to $\pm \infty$ and using a stationary phase approximation. The result is [7]

$$
f_{g}(k, 0)=-i k b_{g} \exp \left[2 i \eta\left(b_{g}\right)-i \pi / 4\right] \sqrt{\frac{\pi}{-\alpha}},
$$

where $b_{g}$ is the impact parameter when the phase shift has a maximum, and

$$
\alpha=\left.\frac{d^{2} \eta}{d b^{2}}\right|_{b=b_{g}} .
$$

The phase shift $\eta\left(b_{1}\right)$ in (12) is usually set equal to a small constant times $\pi$ [7], so that the long-range contribution to the scattering amplitude is linear in $k$ and quadratic in the impact parameter $b_{1}$. Since the glory contribution is linear in $k$ and $b_{g}$, it would appear that it should be dominated by the long-range contribution. In the present study, however, the condition

$$
\left|\eta\left(b_{g}\right)-\eta\left(b_{1}\right)\right| \gg 1
$$

for the stationary phase approximation to be valid breaks down. Therefore, we must use Eq. (14) directly. This can be done through use of the eikonal approximation for the phase shift

$$
\eta(b)=-\frac{1}{4 k} \int_{-\infty}^{+\infty} U(b, z) d z
$$

where

$$
U(b, z)=2 \mu V\left(\sqrt{b^{2}+z^{2}}\right) .
$$

Numerical calculations of (14) reveal that the glory contribution is typically three times larger than the long-range contribution (12).

Further evidence for the importance of glory scattering comes from the molecular potentials themselves. The longrange dispersion energy can generally be found very accurately. Its contribution $V_{L}(r)$ to the potential is given by the large- $r$ van der Waals expansion

$$
V_{L}(r) \sim-\frac{C_{6}}{r^{6}}-\frac{C_{8}}{r^{8}}-\frac{C_{10}}{r^{10}} .
$$

The short-range repulsive part of the potential is mainly due to exchange and electrostatic forces, and is usually approximated by the small- $r$ Born-Mayer potential

$$
V_{S}(r) \sim A \exp (-a r) .
$$

Therefore, probably the most uncertain part of the potential is near the minimum. The glory impact parameter $b_{g}$ is typically found to be located near the position of this minimum. In the next section, we will discuss the sensitivity of the forward scattering amplitude to the uncertainty in the molecular potential in the intermediate range where the minimum occurs.

The actual potentials were obtained semiempirically from the calculations of Bottcher, Dalgarno, and Wright [8] using the functional form

$$
V(r)=V_{S}(r)+V_{L}(r) .
$$

In order to avoid the small- $r$ divergence of the van der Waals expansion (20), weu se

$$
V_{L}(r)=-C_{6} g_{6}(a ; r)-C_{8} g_{8}(a ; r)-C_{10} g_{10}(a ; r),
$$

where

$$
g_{n}(a ; r)=\frac{\gamma(n, a r)}{(n-1) ! r^{n}}
$$

is related to the universal damping function of Tang and Toennies [10]. The incomplete gamma function $\gamma(n, a r)$, defined by

$$
\gamma(n, a r)=(n-1) !\left[1-\exp (-a r) \sum_{m=0}^{n-1} \frac{(a r)^{m}}{m !}\right],
$$

regularizes the short-range behavior of the attractive part of the potential, while allowing for an exact long-range van der Waals expansion. For the short-range potential, we use

$$
V_{S}(r)=\sum_{n} A_{n} \Gamma(n, a r)
$$

The complementary incomplete gamma function $\Gamma(n, a r)$ is used to provide short-range expansions that are more flexible than the Born-Mayer repulsive potential (21). The potentials (26) are analytically equivalent to the Born-Mayer potential multiplied by a polynomial in $r$.

To determine the parameters for our potentials, we used a least squares fit to the calculated data [8]. The long-range van der Waals coefficients are taken from [11] and shown in Table I, while the optimized short-range parameters are shown in Table II. For helium, two different short-range potentials were used, each having a root-mean-square (rms) deviation from the data on the order of $10^{-5}$ (the fits are

TABLE I. List of the long-range coefficents (from Ref. [11].

\begin{tabular}{cccc}
\hline \hline & $C_{6}$ & $C_{8}$ & $C_{10}$ \\
\hline $\mathrm{He}$ & 24.7 & 1320 & 87000 \\
$\mathrm{Ne}$ & 48.0 & 2900 & 190000 \\
\hline \hline
\end{tabular}


TABLE II. List of the short-range parameters.

\begin{tabular}{lccccc}
\hline \hline & $a$ & $A_{1}$ & $A_{2}$ & $A_{3}$ & $A_{4}$ \\
\hline $\mathrm{He}^{(1)}$ & 1.2944 & 5.5111 & -2.1446 & 0.2807 & 0.0000 \\
$\mathrm{He}^{(2)}$ & 1.4725 & 2.2630 & 1.7760 & -0.6261 & 0.0701 \\
$\mathrm{Ne}$ & 1.5800 & 5.3874 & 6.5796 & -1.6062 & 0.1603 \\
\hline \hline
\end{tabular}

denoted by $\mathrm{He}^{(1)}$ and $\mathrm{He}^{(2)}$ in Table II). In general, for a given rms deviation from the data, a fit which includes $A_{4}$ will provide a slightly deeper well than one which constrains $A_{4}$ to be zero. For both of the helium fits, the nonlinear parameter $a$ was determined by the criterion that the rms deviation from the data be minimized. For neon, the choice of $a$ was determined by the requirement that the fitted potential give a dissociation energy that is closest to the spectroscopically known value [9] of5c $\mathrm{m}^{-1}$. Our best fit for neon gave a dissociation energy of $4.58 \mathrm{~cm}^{-1}$. We also used potentials provided by Peach [12]. These potentials contain enough points that we were able to use cubic splines to interpolate the data. The potential obtained from the spline fit for neon gives a dissociation energy of $4.9 \mathrm{~cm}^{-1}$. Figures 1 and 2 show the respective $\mathrm{NaHe}$ and $\mathrm{NaNe}$ interaction potentials in the vicinity of the well.

\section{RESULTS}

In Fig. 3, we show the distribution functions $\rho(k)$ of the relative momentum for collisions in the center of mass frame. For both the helium and neon target gases, the spread is wide enough that the velocity distribution of the sodium projectile can be neglected.

Figure 4 shows the ratio $R$ of the real to the imaginary part of the forward scattering amplitude for helium as a function of the sodium projectile velocity. $R$ is shown for two nonlinear least squares fits to the theoretical potential data of Bottcher, Dalgarno, and Wright [8], for a cubic spline fit to the theoretical potential data of Peach and co-workers [12], and for the experimental measurements of Schmiedmayer et al. [2]. The only noticeable difference between the two

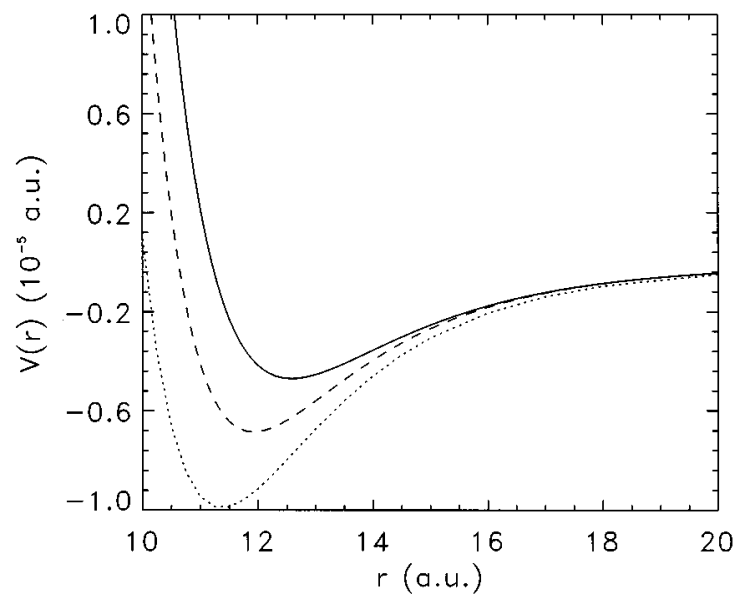

FIG. 1. The interaction potentials for NaHe. The solid and dashed curves are for the nonlinear least squares fits denoted by $\mathrm{He}^{(1)}$ and $\mathrm{He}^{(2)}$ in Table II. The dotted curve is for the cubic spline fit to the data [12].

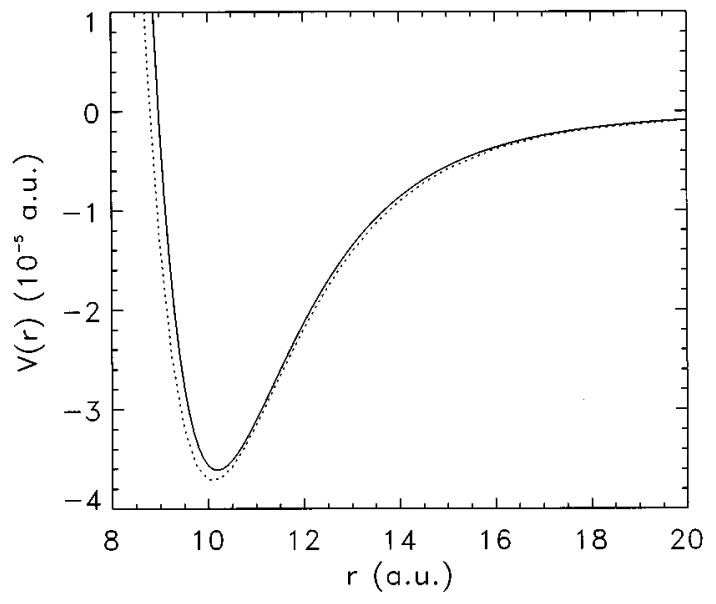

FIG. 2. The interaction potentials for NaNe. The solid curve is for the linear least squares fit in Table II. The dotted curve is for the cubic spline fit to the data [12].

nonlinear fits (see $\mathrm{He}^{(1)}$ and $\mathrm{He}^{(2)}$ in Table II) is the size and location of the potential minimum. The sensitivity of the scattering amplitude to small changes in the potential well is clearly very large. A small increase in the depth of the well causes a large increase in the value of $R$. The experimental data appear to lie in between the calculations using the two nonlinear potential fits. The agreement between the experimental data and the calculation of $R$ using the potential data $[12]$ is not as good. The structure that appears in the experimental data is not reproduced in any of the theoretical calculations, indicating the need for further investigations. However, considering the difficulty of the experiments, the level of agreement is encouraging.

For neon, the results are given in Fig. 5. The potentials obtained from the least squares fit (see Table II) and the cubic spline fit to the data [12] both give a dissociation energy that is close to the measured value [9] of $5 \mathrm{c} \mathrm{m}^{-1}$. The agreement between theory and experiment for neon is excellent, except for velocities less than $800 \mathrm{~m} / \mathrm{s}$ where the experimental uncertainties are large (see Fig. 5).

We have studied theoretically the index of refraction of

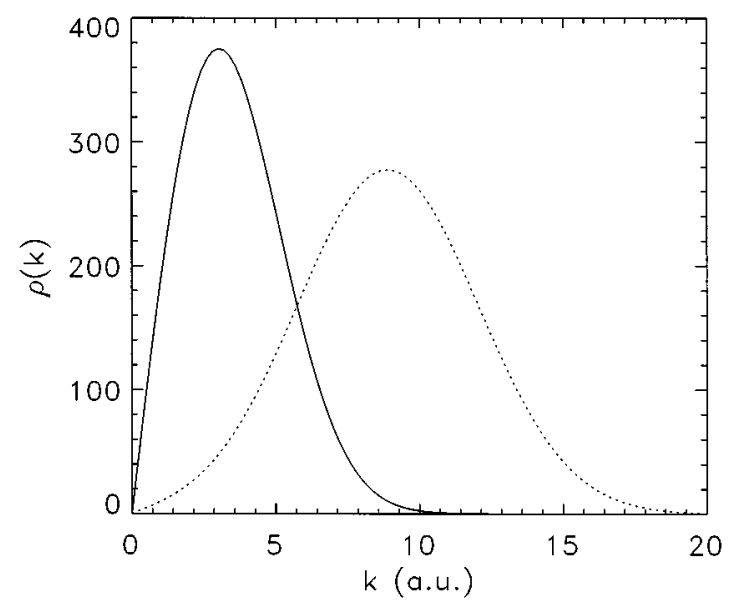

FIG. 3. The distribution of the relative momentum for the collisions in the center of mass frame. The projectile velocity is 1000 $\mathrm{m} / \mathrm{s}$ and the temperature of the gas is $300 \mathrm{~K}$. The solid curve is for $\mathrm{He}$ and the dotted curve is for $\mathrm{Ne}$. 


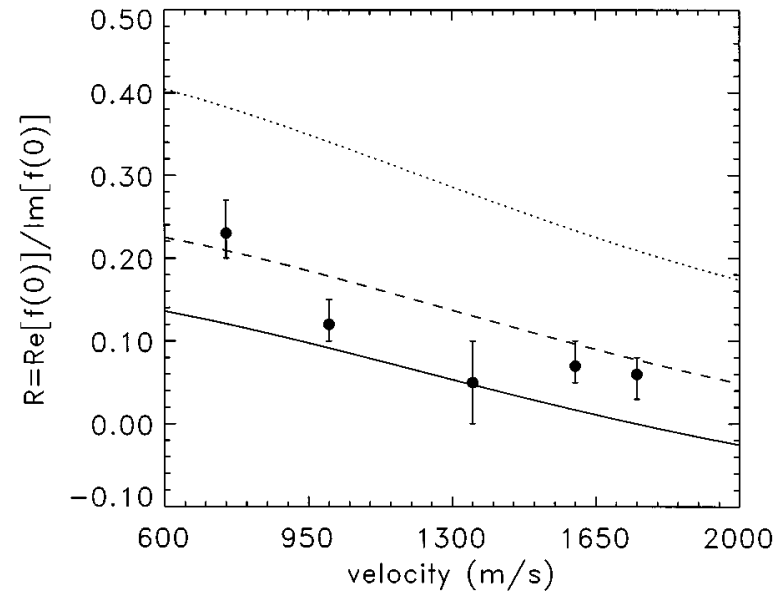

FIG. 4. The ratio of the real to the imaginary part of the forward scattering amplitude for He. The solid and dashed curves are for the nonlinear least squares fits denoted by $\mathrm{He}^{(1)}$ and $\mathrm{He}^{(2)}$ in Table II. The dotted curve is for the cubic spline fit to the data [12], and the points with error bars are for the experimental data [2].

the noble gases $\mathrm{He}$ and $\mathrm{Ne}$ for the propagation of the sodium matter waves. We find that our numerical calculations agree well with the data from the interferometer experiment [2]. Our calculations provide an interpretation for the experimental data, and may be useful in selecting the best interatomic potentials. For example, the atom interferometry measurements shown in Fig. 4 indicate that the exact $\mathrm{NaHe}$ potential probably lies between the solid and dashed curves shown in Fig. 1. In contrast to the interpretation presented in [2], we have argued that the two-body forward scattering amplitude is dominated by the so-called "glory" contribution. Because of the important role played by the glory scattering, the measured ratio of the real to the imaginary part of the forward scattering amplitude is very sensitive to the intermediaterange of the molecular potential curves. With accurate longrange coefficients $C_{6}, C_{8}$, and $C_{10}$, and short-range information about the repulsive wall, it should be possible to infer

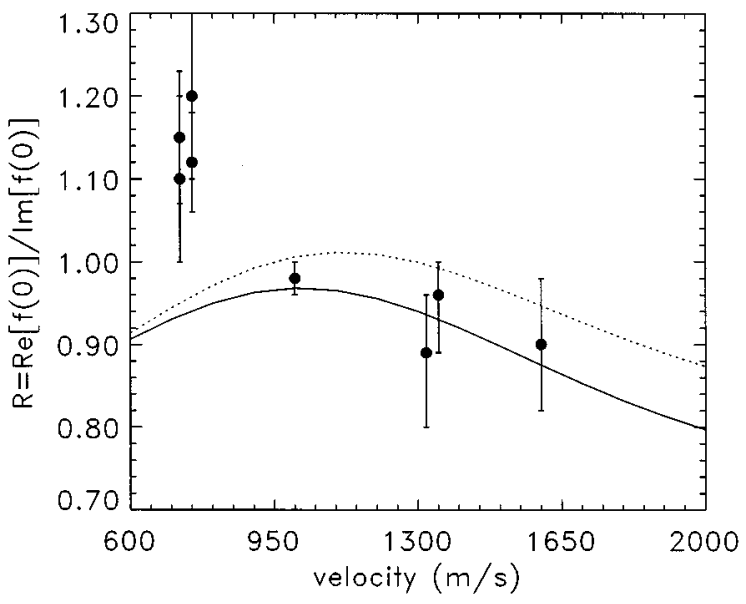

FIG. 5. The ratio of the real to the imaginary part of the forward scattering amplitude for Ne. The solid curve is for the linear least squares fit in Table II. The dotted curve is for the cubic spline fit to the data [12], and the points with error bars are for the experimental data [2].

from the measured ratio the correct intermediate-range potential parameters, such as the potential well depth.

\section{ACKNOWLEDGMENTS}

The authors gratefully acknowledge Yan Sun for many helpful communications. We also thank Gillian Peach for providing her most recent potential calculations, and David Pritchard and members of his MIT atom interferometer group for helpful discussions and for providing the experimental data used in this work. This work was supported by the U.S. Department of Energy, Division of Chemical Sciences, Office of Basic Energy Sciences, Office of Energy Research, and by the National Science Foundation through a grant for the Institute for Theoretical Atomic and Molecular Physics at Harvard University and Smithsonian Astrophysical Observatory.
[1] For a recent review, see Appl. Phys. B 54 (1992), special issue on Optics and Interferometry with Atoms, edited by J. Mlynek, V. Balykin, and P. Meystre.

[2] J. Schmiedmayer, M. S. Chapman, C. R. Ekstrom, T. D. Hammond, S. T. Wehinger, and D. E. Pritchard, Phys. Rev. Lett. 74, 1043 (1995); C. R. Ekstrom, Ph.D. thesis, MIT, 1993.

[3] D. W. Keith, C. R. Ekstrom, Q. A. Turchette, and D. E. Pritchard, Phys. Rev. Lett. 66, 2693 (1991).

[4] V. F. Sears, Phys. Rep. 82, 1 (1982).

[5] R. J. Glauber, in Lectures in Theoretical Physics, edited by W. E. Brittin and L. G. Dunham (Interscience, New York, 1959), Vol. I, p. 315.

[6] M. Lax, Rev. Mod. Phys. 23, 287 (1951).
[7] B. H. Bransden and M. R. C. McDowell, Charge Exchange and the Theory of Ion-Atom Collisions (Oxford University Press, New York, 1992).

[8] C. Bottcher, A. Dalgarno, and E. L. Wright, Phys. Rev. A 7, 1606 (1973).

[9] R. A. Gottscho, R. A.-Bitar, W. P. Lapatovich, I. Renhorn, and D. E. Pritchard, J. Chem. Phys. 75, 2546 (1981).

[10] K. T. Tang and J. P. Toennies, J. Chem. Phys. 80, 3726 (1984).

[11] A. A. Radzig and B. M. Smirnov, Reference Data on Atoms, Molecules, and Ions (Springer-Verlag, Berlin, 1985).

[12] For NaNe, we used the data from C. R. Mason, Ph.D. thesis, University of London, 1991; for $\mathrm{NaHe}$, we used the data from P. J. Leo and G. Peach (unpublished). 